\title{
ASSOCIATED AND SKEW-ORTHOLOGIC SIMPLEXES
}

\author{
BY
}

\author{
LEON GERBER
}

\begin{abstract}
ABSTRACr. A set of $n+1$ lines in $n$-space is said to be associated if every $(n-2)$-flat which meets $n$ of the lines also meets the remaining line. Two simplexes are associated if the joins of their corresponding vertices are associated. Two simplexes are (skew-)orthologic if the perpendiculars from the vertices of one on the faces of the other are concurrent (associated); it follows that the reciprocal relation holds. In an earlier paper, Associated and Perspective Simplexes, we gave an affine necessary and sufficient condition for two simplexes to be associated that was so easy to apply that extensions to $n$-dimensions of nearly all known theorems, and a few new ones, were proved in a few lines of calculations. In this sequel we take a closer look at some of the results of the earlier paper and prove some new results. Then we give simple Euclidean necessary and sufficient conditions for two simplexes to be orthologic or skew-orthologic which yield as corollaries known results on altitudes, the Monge point and orthocentric simplexes. We conclude by discussing some of the qualitative differences between the geometries of three and higher dimensions.
\end{abstract}

1. Introduction. Let $\mathscr{Q}$ and $\mathscr{B}$ be $n$-simplexes with vertices and faces $A_{i}, B_{i}, \mathbb{Q}_{i}, \mathscr{B}_{i}$, respectively, $i \in \mathcal{G}$, where $\mathcal{G}=\{0,1, \ldots, n\}$. We say that $\mathbb{Q}$ and $\mathscr{B}$ are associated if the lines $A_{i} B_{i}$ joining corresponding vertices are associated, i.e. have the property that every secundum $((n-2)$-flat $)$ which meets $n$ of the lines also meets the remaining line. For $n=2$ the last statement reduces to: any point which lies on two of the lines lies on the third. Thus the notion of associated simplexes is a generalization of that of perspective simplexes. In a recent paper [6], we gave an affine necessary and sufficient condition for two simplexes to be associated and gave a very short proof of extensions to $n$-dimensions of many theorems from Neuberg's famous Memoir sur le Tétraèdre as well as some new results. In $\$ \S 2$ and 3 of this sequel we examine the significance of certain parameters we introduced and give a family of constructions which lead to pairs of associated simplexes.

Received by the editors August 19, 1975.

AMS (MOS) subject classifications (1970). Primary 50B99; Secondary 50B10, 50D05.

Key words and phrases. Simplex, perspective simplexes, associated simplexes, orthologic simplexes, skew-orthologic simplexes. 
In $\S 4$ we give an extension to $n$-dimensions of the only known result on associated lines which is truly affine.

In the same Memoir [15, pp. 12, 27]. Neuberg showed that if the perpendiculars from the vertices of one tetahedron on the faces of a second are concurrent or associated, the reciprocal relation also holds. He called such tetrahedra respectively orthologic and skew-orthologic. We give here in $\$ 5$ new and simple necessary and sufficient conditions for two simplexes to be orthologic or skew-orthologic which, though they are Euclidean, bear a striking resemblance to the earlier affine criteria for associativity. At the same time these conditions exhibit a duality relation between the cosines of dihedral angles and the exponentials of the edge-lengths-squared previously found only in non-Euclidean geometry. In $\$ 6$ we conclude with some remarks to the effect that the step from three to $n$ dimensions may hold as many surprises as the step from two to three dimensions.

We use the same notation as in [6] and Theorem i.j of [6] will be called Fact i.j here.

2. Composite and semisymmetric matrices. Let the weights (barycentric coordinates) of $B_{i}$ with respect to $Q$ be given by $B_{i}=\left(b_{i 0}, b_{i 1}, \ldots, b_{i n}\right)=\left(b_{i j}\right)$, $i \in 9$, and let us call the matrix $\left(b_{i j}\right)$ composite if there exist numbers $y_{i}, z_{i}, i \in \mathcal{G}$, such that $b_{i j}=y_{i} z_{j}$ for $i \neq j$, or, for some $i \in \mathcal{G}$, all elements not in row $i$, column $i$, or the main diagonal, are zero.

The following combination of Facts 3.1 and 4.1 gives the complete significance of the numbers $y_{i}$ and $z_{i}$ for perspective simplexes.

THEOREM 2.1. Let the weights of $B_{i}$ with respect to $\mathbb{Q}$ be $\left(b_{i j}\right)$ and let $s_{i}=\Sigma_{j} b_{i j}, i \in 9$. The following statements are equivalent.

.1. $\left(b_{i j}\right)$ is composite with $b_{i j}=y_{i} z_{j}, i \neq j$. If $s=\sum z_{j}$ is not zero, we may assume $s=1$.

.2. The lines $A_{i} B_{i}, i \in 9$, are concurrent at the point $P=\left(z_{i}\right)$, i.e. $\mathcal{Q}$ and $\mathscr{B}$ are perspective from the point $P$.

.3. The secunda $\mathbb{Q}_{i} \cap \mathscr{B}_{i}, i \in \mathcal{G}$, lie in the prime $\mathscr{F}$, where $\mathscr{F}=\left(y_{i}\right)$ with respect to $\mathscr{B}$ and $\mathscr{F}=\left(y_{i} /\left(s_{i}-s y_{i}\right)\right)$ with respect to $\mathbb{Q}$, i.e. $\mathbb{Q}$ and $\mathscr{B}$ are perspective from the prime $F$.

.4. Ceva's form. For all triples of distinct subscripts,

$$
b_{i j} b_{j k} b_{k i}=b_{j i} b_{k j} b_{i k}
$$

Proof. The equivalence of .1 and .4 is an elementary exercise in algebra. The equivalence of conditions $.1, .2$ and .3 is established in Facts 3.1 and 4.1 except for the assertions regarding the weights. (The proof of Fact 3.1 tacitly assumes that $P$ is distinct from $A_{i}$; the additional clause in the definition given 
here is easily seen to correspond to the case $P=A_{i}$.) In the proof of Fact 3.1 we have computed the weights of $P$ and seen that

$$
\mathbf{b}_{i}=\left(s_{i}-s y_{i}\right) \mathbf{a}_{i}+y_{i} \mathbf{p}
$$

Thus

$$
y_{j} \mathbf{b}_{i}-y_{i} \mathbf{b}_{j}=y_{j}\left(s_{i}-s y_{i}\right) \mathbf{a}_{i}-y_{i}\left(s_{j}-s y_{j}\right) \mathbf{a}_{j}
$$

which shows that lines $B_{i} B_{j}$ and $A_{i} A_{j}$ intersect on the prime $\mathscr{F}$ with weights as given. This completes the proof.

The following dual may also be proved.

THEOREM 2.2. If $\mathfrak{B}_{i}=\left(b_{i j}\right)$ and $b_{i j}=y_{i} z_{j}, i \neq j$, then the $\left(y_{i}\right)$ may be chosen to be the weights of the center and the $z_{i}$ the weights of the prime of perspectivity.

We call the matrix $\left(b_{i j}\right)$ semisymmetric if we can find parameters $x_{i}, i \in \mathcal{G}$, not all zero such that $x_{i} b_{i j}=x_{j} b_{j i}, i, j \in 9$. It is easily seen that a composite matrix is semisymmetric with parameters $x_{i}=z_{i} / y_{i}$. It is an exercise in algebra to show that for $n \geqslant 3,\left(b_{i j}\right)$ is semisymmetric if and only if

$$
b_{i j} b_{k l}=b_{i l} b_{k j} \text { for all quadruples of distinct subscripts. }
$$

We recall

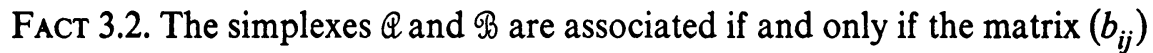
is semisymmetric.

To interpret the parameters $x_{i}$ we introduce the equicenter [16] of two simplexes $\mathbb{Q}$ and $\mathscr{B}$ : the point (in general unique) whose weights with respect to $\mathscr{Q}$ and $\mathscr{B}$ are equal. The equicenter may also be viewed as the invariant point of the affinity which maps $\mathbb{Q}$ into $\mathscr{B}$. There are also, in general, $n$ vectors, which we shall call equivectors, with the same property.

THEOREM 2.3. Let $\mathbb{Q}$ and $\mathscr{B}$ be associated simplexes such that $x_{i} b_{i j}=x_{j} b_{j i}$, for constants $x_{i}, i \in 9$. If $\Re$ is proper then the $x_{i}$ are proportional to the weights of the equicenter of $\mathscr{Q}$ and $\oiint$.

Proof. Let $P$ be the point whose weights with respect to $B$ are (proportional to) $\left(x_{i}\right)$. Then

$$
\mathbf{p}=\sum_{i} x_{i} \mathbf{b}_{i}=\sum_{i} x_{i} \sum_{j} b_{i j} \mathbf{a}_{j}=\sum_{j} x_{j}\left(\sum_{i} b_{j i}\right) \mathbf{a}_{j}=\sum_{j} x_{j} \mathbf{a}_{j}
$$

so the weights of $P$ with respect to $\mathbb{Q}$ are also $\left(x_{j}\right)$.

3. Some special pairs of associated simplexes. The diagonal elements of a matrix $B$ are of no concern in deciding if $B$ is semisymmetric or composite, and they are often of a special form, e.g. the $i$ th weight of $B_{i}$ is zero if $B_{i} \in \mathbb{Q}_{i}$. 
In such a case we shall place them after a colon so that, for example, $A_{i}=(0: 1)$ means that the $i$ th weight is 1 and the remaining weights are zero.

The following result gathers the representations we shall need for our first theorem. Let $v_{k}$ denote the $k$-dimensional volume function and let $v=v_{n}(\mathbb{Q})$. For $i \in g$, let $f_{i}=v_{n-1}\left(\mathbb{Q}_{i}\right), c_{i j}=\cos \left(\mathbb{Q}_{i}, \mathbb{Q}_{j}\right)=c_{j i}$. Let $g^{\prime}=g-\{0\}$ and let $\Sigma, \Sigma^{\prime}, \Sigma^{(i)}$ have ranges $\mathscr{G}, \mathcal{G}^{\prime}$ and $g-\{i\}$, respectively.

LEMMA 3.1. Let $Q$ be an $n$-simplex.

.1. The centroid of $Q$ is given by $G=(1 /(n+1))$.

.2. The centroid of the face $Q_{i}$ is given by $G_{i}=(1 / n: 0), i \in 9$.

3. The foot of the altitude from $A_{i}$ is given by $H_{i}=\left(f_{i}^{-1} f_{j} c_{i j}: 0\right)$, and $\sum_{j}^{(i)} f_{j} c_{i j}=f_{i}$.

.4. The altitude $\overrightarrow{A_{i} H_{i}}=\left(f_{i}^{-1} f_{j} c_{i j}:-1\right)$.

.5. A unit vector $\mathbf{n}_{i}$ normal to $\mathbb{Q}_{i}$ is given by $n v \mathrm{~h}_{i}=f_{i} \overrightarrow{A_{i} H_{i}}=\left(f_{j} c_{i j}:-f_{i}\right)$.

.6. The vector hyperarea of $\mathcal{Q}_{i}$ is given by $\mathbf{f}_{i}=f_{i} \mathbf{n}_{i}=(1 / n v)\left(f_{i} f_{j} c_{i j}:-f_{i}^{2}\right)$.

7. The incenter of $\mathfrak{Q}$ is given by $T=\left(f_{j} / f\right)$ where $f=\sum f_{j}$ and the inradius, $r$, is given by $r f=\sum r f_{j}=n v$.

8. The incenter of $\mathbb{Q}_{i}$ is given by $\left(f_{i j} / f^{i}: 0\right)$ where $f_{i j}=v_{n-2}\left(Q_{i j}\right)$ and $f^{i}$ $=\sum_{j}^{(i)} f_{i j}$, and its inradius $r^{i}$ is given by $r^{i} f^{i}=(n-1) f_{i}$.

.9. The center of the ith exsphere (the sphere tangent to $\mathfrak{Q}_{i}$ externally and to $\mathfrak{Q}_{j}$ internally, $j \neq i)$ is given by $T_{i}=\left(f_{j} /\left(f-2 f_{i}\right):-f_{i} /\left(f-2 f_{i}\right)\right)$ and its radius $r_{i}$ is given by $r_{i}\left(f-2 f_{i}\right)=\sum_{j}^{(i)} r_{i} f_{j}-r_{i} f_{i}=n v$.

.10. The point of contact of the ith exsphere with $\mathscr{Q}_{i}$ is given by $\mathbf{t}_{i}-r_{i} \mathbf{n}_{i}$ $=\left(f_{j}\left(1-c_{i j}\right) /\left(f-2 f_{i}\right): 0\right)$.

.11. The Lemoine point of $\mathscr{Q}$ is given by $K=\left(f_{i}^{2} / f^{\prime \prime}\right)$ where $f^{\prime \prime}=\Sigma f_{i}^{2}$.

Proof. Projecting $A_{i}$ orthogonally on $\mathbb{Q}_{i}$ gives .3 immediately. Proofs of .7, .8 , and .9 are found in [5]. Result .11 is Fact 7.1 and the remaining results are easily seen to be true.

THEOREM 3.2. Let $\mathbb{Q}$ be an $n$-simplex. For $i \in \mathfrak{G}$, let the points $B_{i}$ be determined by $\vec{C}_{i} \vec{B}_{i}=x v_{i}$ where $x$ is an arbitrary number and $C_{i}$ and $v_{i}$ are the points and vectors given by any one of conditions .1-.8 below. Then $\mathbb{Q}$ and $\mathscr{B}$ are associated.

.1. $C_{i}$ is the centroid $G$ of $\mathbb{Q}$ and $\mathbf{v}_{i}$ is the hyperarea $\mathbf{f}_{i}$.

.2. $C_{i}$ is the centroid of $\mathbb{Q}_{i}$ and $\mathbf{v}_{i}$ is the hyperarea $\mathbf{f}_{i}$.

.3. $C_{i}$ is the incenter of $\mathscr{Q}$ and $\mathbf{v}_{i}$ is the unit normal $\mathbf{n}_{i}$.

.4. $C_{i}$ is the incenter of $\mathbb{Q}_{i}$ and $\mathrm{v}_{i}=r^{i} \mathbf{n}_{i}$.

.5. $C_{i}$ is the ith excenter and $\mathbf{v}_{i}=r_{i} \mathbf{n}_{i}$.

.6. $C_{i}$ is the point of contact of the insphere with $\mathscr{Q}_{i}$, and $\mathbf{v}_{i}=\mathbf{n}_{i}$.

.7. $C_{i}$ is the point of contact of the ith exsphere with $Q_{i}$, and $\mathbf{v}_{i}=r_{i} \mathbf{n}_{i}$.

.8. $C_{i}$ is the Lemoine point of $\mathcal{Q}$ and $\mathrm{v}_{i}=\overrightarrow{A_{i} H_{i}}$.

Proof. We shall assume that the constant $n v$ is absorbed in $x$. 
In .1 we have $\mathbf{b}_{i}=\mathbf{g}+x \mathbf{f}_{i}$ so $B_{i}=\left((1 / n+1)+x f_{i} f_{j} c_{i j}:(1 / n+1)-x f_{i}^{2}\right)$ and $b_{i j}=b_{j i}$ for $i \neq j$, so $\mathbb{Q}$ and $\mathscr{B}$ are associated from Fact 3.2.

In the remaining parts we give $b_{i j}$ for $i \neq j$ and the value of $x_{i}$ so that $x_{i} b_{i j}=x_{j} b_{j i}$.

.2. $b_{i j}=(1 / n)+x f_{i} f_{j} c_{i j}, x_{i}=1$.

3. $b_{i j}=f_{j} / f+x f_{j} c_{i j}, x_{i}=f_{i}$.

.4. $b_{i j}=f_{i j} / f^{i}+x\left(f_{i} / f^{i}\right) f_{j} c_{i j}, x_{i}=f^{i}$.

.5. $b_{i j}=f_{j} /\left(f-2 f_{i}\right)+x f_{j} c_{i j} /\left(f-2 f_{i}\right), x_{i}=f_{i}\left(f-2 f_{i}\right)$.

.7. $b_{i j}=f_{j}\left(1-c_{i j}\right) /\left(f-2 f_{i}\right)+x f_{j} c_{i j} /\left(f-2 f_{i}\right), x_{i}=f_{i}\left(f-2 f_{i}\right)$.

.8. $b_{i j}=f_{j}^{2} / f^{\prime \prime}+x f_{i}^{-1} f_{j} c_{i j}, x_{i}=f_{i}^{2}$.

To prove .6 we need only apply .3 with $x$ increased by the inradius $r$, which completes the proof. Theorem 3.2.2 is a generalization of the theorem [23, Theorem 5] that the outer or inner Napoleon triangle of triangle $\mathscr{Q}$ (the centers of equilateral triangles constructed outward or inward on the sides of $\mathbb{Q}$ ) is perspective with $\mathbb{Q}$. The three dimensional version of Theorem 3.2.4 is [18, p. 61]. Applying the remark after Theorem 2.2, the following is clear.

COROLLARY 3.3. The pairs of simplexes in parts .1 and .2 above have a common centroid. The equicenter of the simplexes of parts .3 and .6 is the incenter of $Q$ and the equicenter of the simplexes of part .8 is the Lemoine point of $\mathbb{Q}$.

By setting $x=0$ in various parts of Theorem 3.2, we obtain:

COROLlary 3.4.

.1. [FACT 6.7] The lines joining each vertex $A_{i}$ to the incenter of $\mathbb{Q}_{i}, i \in \mathscr{G}$, are associated.

.2. [FACT 6.6] The lines joining each vertex $A_{i}$ to the point of contact of the insphere with $\mathbb{Q}_{i}, i \in \mathcal{G}$, are associated.

.3. $\left[15\right.$, p. 26] The lines joining each vertex $A_{i}$ to the point of contact of the ith exsphere with $\mathbb{Q}_{i}, i \in \mathcal{G}$, are associated.

The perpendiculars to the faces of $\mathbb{Q}$ at their centroids are the images of the altitudes in the dilation with center at the centroid and constant $-1 / n$ so they are associated. Thus the simplexes constructed in Theorem 3.2.2 are skeworthologic as well as associated.

4. An affine property. The first part of the next result is obvious; the second part, of which a strictly three dimensional proof is given in [4], is quite surprising.

THEOREM 4.1. Let $\mathscr{F}$ and $\mathcal{G}$ be two parallel primes, let the line $\mathfrak{L}_{0}$ meet $\mathscr{F}$ and $\mathcal{G}$ in $B_{0}$ and $A_{0}$ respectively, and, for $i \in \mathcal{G}^{\prime}$, let the lines $\mathfrak{L}_{i}$ meet $\mathscr{F}$ and $\mathcal{S}$ in $A_{i}$ and $B_{i}$ respectively. The weights of $B_{0}$ with respect to $\mathfrak{Q}_{0}$ are the same as the weights 
of $A_{0}$ with respect to $\mathscr{B}_{0}$ if either of the following conditions hold.

.1. The lines $\mathfrak{L}_{i}$ concur at a point $P$.

.2. The lines $\mathcal{L}_{i}$ are merely associated.

Proof. Condition .1 implies that the points of $\mathscr{F}$ are related to the points of $\mathcal{S}$ by a dilation with center $P$ and the result is immediate.

Suppose we have only condition .2 . With respect to the simplex $\mathbb{Q}$, let $B_{i}=\left(b_{i j}\right), i \in 9$. Then since $B_{0}$ lies in $A_{0}$, and for $i \in \mathcal{G}^{\prime}, B_{i}$ lies in a prime through $A_{0}$ parallel to $A_{i}$ and since the sum of the weights of a proper point is 1 , we have

$$
b_{00}=0, \quad \Sigma^{\prime} b_{0 j}=1, \quad b_{i 0}=1 \text { and } \sum_{j}^{\prime} b_{i j}=0 \text { for } i \in g^{\prime} .
$$

Since the lines are associated there exist numbers $x_{i}, i \in \mathcal{G}$, not all zero such that $x_{i} b_{i j}=x_{j} b_{j i}, i \neq j$. In particular, $x_{0} b_{0 j}=x_{j} b_{j 0}=x_{j}, j \in q^{\prime}$, so $x_{0} \neq 0$ and we may take $x_{0}=1$. Thus

$$
b_{0 j} b_{j i}=b_{0 i} b_{i j}
$$

Now using (4.1) and (4.2) we find

$$
\begin{aligned}
\sum_{j}^{\prime} b_{0 j} \mathbf{b}_{j} & =\sum_{j}^{\prime} b_{0 j}\left[\mathbf{a}_{0}+\sum_{i}^{\prime} b_{j i} \mathbf{a}_{i}\right] \\
& =\left(\sum_{j}^{\prime} b_{0 j}\right) \mathbf{a}_{0}+\sum_{i}^{\prime}\left(\sum_{j}^{\prime} b_{0 j} b_{j i}\right) \mathbf{a}_{i} \\
& =\mathbf{a}_{0}+\sum_{i}^{\prime} b_{0 i}\left(\sum_{j}^{\prime} b_{i j}\right) \mathbf{a}_{i}=\mathbf{a}_{0}
\end{aligned}
$$

which proves the result.

Corollary 4.2. For $i \in \mathfrak{G}$, let $\mathfrak{L}_{i}$ and $\mathfrak{N}_{i}$, be two sets of associated lines such that $\mathfrak{L}_{i}$ is parallel to $\Re_{i}$. Let the prime $\mathscr{F}$ meet $\mathfrak{L}_{i}$ in $A_{i}$ and $\Re_{i}$ in $B_{i}, i \in \mathcal{G}$. If $\mathbf{a}_{0}=\Sigma^{\prime} w_{i} \mathbf{a}_{i}$, then $\mathbf{b}_{0}=\Sigma^{\prime} w_{i} \mathbf{b}_{i}$.

Proof. Let the prime $\mathcal{G}$, distinct from and parallel to $\mathscr{F}$, meet $\mathscr{L}_{i}$ in $A_{i}^{\prime}$ and $\Re_{i}$ in $B_{i}^{\prime}, i \in g$. Then $\overrightarrow{A_{0} A_{0}^{\prime}}=\mathbf{a}_{0}^{\prime}-\mathbf{a}_{0}=\sum^{\prime} w_{i} \mathbf{a}_{i}^{\prime}-\Sigma^{\prime} w_{i} \mathbf{a}_{i}=\Sigma^{\prime} w_{i} \overrightarrow{A_{i} A_{i}}$. Since $\overrightarrow{B_{i} B_{i}^{\prime}}=\overrightarrow{A_{i} A_{i}^{\prime}}, i \in \mathcal{G}$, we have $\overrightarrow{B_{0} B_{0}^{\prime}}=\Sigma^{\prime} w_{i} \overrightarrow{B_{i} B_{i}^{\prime}}$ and hence $b_{0}$ $=\Sigma^{\prime} w_{i} \mathbf{b}_{i}$.

This theorem and corollary are remarkable in that all other results on associated lines that we have seen which are not strictly projective require Euclidean notions, whereas these results are truly affine.

5. Orthologic and skew-orthologic simplexes. It was established in the proof of Fact 5.2 that $\mathbb{Q}$ and $\mathscr{B}$ are orthologic or skew-orthologic according as the 
matrix $\left(\cos \left(\mathscr{Q}_{i}, \mathfrak{B}_{j}\right)\right)$ is composite or semisymmetric. We shall derive here some other characterizations in terms of the squares of the distances between the vertices. One of these is particularly easy to apply viz: $\mathbb{Q}$ and $\mathscr{B}$ are orthologic or skew-orthologic according as the matrix $\left(\exp \left(A_{i} B_{j}^{2}\right)\right)$ is composite or semisymmetric. In the orthologic case this condition was discovered by Darboux [19] but in the skew-orthologic case the characterization is believed to be new.

We first derive an intermediate condition. Let ()$,()^{\prime}$, and ()$^{+}$denote row, column or square matrices, depending on context, with subscripts in $g, g^{\prime}$, and $G_{+}=\{0,1, \ldots, n+1\}$ respectively. Define

$$
d_{n}(Q, \mathscr{B})=\left|\begin{array}{cc}
\left(A_{i} B_{j}^{2}\right) & (1) \\
(1) & 0
\end{array}\right|=\left|\begin{array}{ccc}
A_{0} B_{0}^{2} \cdots A_{0} B_{n}^{2} & 1 \\
\cdots & \\
A_{n} B_{0}^{2} \cdots A_{n} B_{n}^{2} & 1 \\
1 \cdots 1 & 0
\end{array}\right|
$$

and let $d_{i j}$ be the minor of $A_{i} B_{j}^{2}, i, j \in 9$.

THEOREM 5.1. $\mathfrak{Q}$ and $\mathfrak{B}$ are orthologic or skew-orthologic according as $\left(d_{i j}\right)$ is composite or symmetric.

Proof. It is clear that $d_{i j}=d_{n-1}\left(\mathbb{Q}_{i}, \mathscr{B}_{j}\right)$ and it follows from [19, p. 361] that

$$
d_{i j}=(-1)^{n}[(n-1) !]^{2} 2^{n-1} v_{n-1}\left(\mathscr{Q}_{i}\right) v_{n-1}\left(\mathscr{B}_{j}\right) \cos \left(\mathscr{Q}_{i}, \mathscr{B}_{j}\right)
$$

so that $\left(d_{i j}\right)$ has the same property as $\left(\cos \left(\mathscr{Q}_{i}, \mathfrak{B}_{j}\right)\right)$. We shall see later that in the skew-orthologic case $\left(d_{i j}\right)$ is in fact symmetric.

The next theorem collects the main characterization of orthologic simplexes; conditions . 3 and .4 are found in [19, pp. 362-363]; condition .4 is also found in [20].

THEOREM 5.2. Let $\mathscr{Q}$ and $\mathfrak{B}$ be $n$-simplexes. The following are equivalent.

.1. $\mathcal{Q}$ and $\mathcal{B}$ are orthologic.

.2. There exist constants $y_{i}, z_{i}, i \in \mathscr{G}$, such that $\cos \left(\mathbb{Q}_{i}, \mathscr{S}_{j}\right)=y_{i} z_{j}, i \neq j$, i.e. $\left(\cos \left(Q_{i}, \mathscr{T}_{j}\right)\right)$ is composite.

.3. There exist constants $a_{i}, b_{i}, i \in \mathcal{G}$, such that $A_{i} B_{j}^{2}=a_{i}+b_{j}, i \neq j$, i.e. $\left(\exp \left(A_{i} B_{j}^{2}\right)\right)$ is composite.

.4. (For $n \geqslant 3$.) For all quadruples of distinct subscripts, $A_{i} A_{j}$ is perpendicular to $B_{k} B_{1}$, i.e. $A_{i} B_{1}^{2}-A_{i} B_{k}^{2}+A_{j} B_{k}^{2}-A_{j} B_{1}^{2}=0$.

Proof. The equivalence of .1 and .2 was recalled in the preceding proof. If .1 is true, let $A_{+}$be the intersection of the perpendiculars from the vertices of $\mathbb{Q}$ to the faces of $\mathfrak{B}$. Then

(5.1) $0=2 \overrightarrow{A_{i} A_{+}} \cdot \overrightarrow{B_{j} B_{k}}=A_{i} B_{k}^{2}-A_{i} B_{j}^{2}+A_{+} B_{j}^{2}-A_{+} B_{k}^{2}, \quad i, j, k$ distinct. 
For $i \in g$, let $b_{i}=B_{i} A_{+}^{2}$ and $a_{i}=A_{i} B_{i+1}^{2}-b_{i+1}$, where $b_{n+1}=b_{0}$. Clearly, $A_{i} B_{i+1}^{2}=a_{i}+b_{i+1}$. If $k \neq i$ or $i+1$, using $j=i+1$ in (5.1) we have

$$
A_{i} B_{k}^{2}=A_{i} B_{i+1}^{2}-A_{+} B_{i+1}^{2}+A_{+} B_{k}^{2}=\left(a_{i}+b_{i+1}\right)-b_{i+1}+b_{k}=a_{i}+b_{k} .
$$

A simple calculation will show that .3 implies

$$
\begin{aligned}
d_{i j} & =-\prod_{k \neq i, j}\left(A_{k} B_{k}^{2}-a_{k}-b_{k}\right) \\
& =-\left(A_{i} B_{i}^{2}-a_{i}-b_{i}\right)^{-1}\left(A_{j} B_{j}^{2}-a_{j}-b_{j}\right)^{-1} \Pi\left(A_{k} B_{k}^{2}-a_{k}-b_{k}\right)
\end{aligned}
$$

which is clearly composite and implies .1. It is easily seen that .3 is equivalent to .4 which concludes the proof. For $n=2$ the appropriate equivalent of .4 is given by Theorem 5.7.2.

It is useful to regard an orthocentric simplex $\mathscr{Q}$ as the special case of orthologic simplexes $\mathbb{Q}$ and $\mathscr{B}$ where $\mathscr{B}$ coincides with $\mathscr{Q}$. If we denote the orthocenter by $A_{+}$and accept " + " as an abbreviation for " $n+1$ " it is clear that any $n+1$ points of the $n+2$ points $A_{i}, i \in g_{+}$may be regarded as an orthocentric simplex with the remaining point as orthocenter. Such a set of $n+2$ points may be called an orthocentric dupoint.

Similarly for orthologic simplexes $\mathscr{Q}$ and $\mathscr{B}$, let $B_{+}$be the point of intersection of the perpendiculars from $B_{i}$ on $\mathbb{Q}_{i}, i \in \mathfrak{g}$.

It is clear that any $n+1$ pairs of points from the $n+2$ pairs $A_{i}, B_{i}, i \in g_{+}$, are the vertices of orthologic simplexes with the remaining points as centers. We shall call these sets of $n+2$ points orthologic dupoints. If we choose $a_{+}=0$ and $b_{+}=A_{0} B_{+}^{2}-a_{0}$, it is also clear that the equations in .3 and .4 are valid with subscripts in $g_{+}$. The parameters are not unique since 3 remains valid if all the $a_{i}$ are increased and the $b_{i}$ decreased by the same amount. We observe the following properties of the parameters.

THEOREM 5.3. Let $A_{i}, B_{i}, a_{i}, b_{i}, i \in \mathscr{G}_{+}$, be the points and parameters of two orthologic dupoints in $n$-space. Then

.1. $\Sigma^{+}\left(A_{i} B_{i}^{2}-a_{i}-b_{i}\right)^{-1}=0$.

.2. The weights of $A_{+}$with respect to $\mathbb{Q}_{+}$equal those of $B_{+}$with respect to $\mathscr{B}_{+}$and are proportional to $\left(\left(A_{i} B_{i}^{2}-a_{i}-b_{i}\right)^{-1}\right)$.

PROof. Since each set of $n+2$ points lies in $n$-space we have

$$
\begin{aligned}
0 & =d_{n+1}\left(A_{0} \cdots A_{+}, B_{0} \cdots B_{+}\right)=\left|\begin{array}{cc}
\left(a_{i}+b_{j}: A_{i} B_{i}^{2}\right)^{+} & (1)^{+} \\
(1)^{+} & 0
\end{array}\right| \\
& =\left|\begin{array}{cc}
\left(0: A_{i} B_{i}^{2}-a_{i}-b_{i}\right)^{+} & (1)^{+} \\
(1)^{+} & 0
\end{array}\right|
\end{aligned}
$$


after subtracting $a_{i}$ (respectively $b_{j}$ ) times the last row (column) from row $i$ (column $j$ ). After dividing row $i$ by $A_{i} B_{i}^{2}-a_{i}-b_{i}$ and subtracting from the last row we obtain

$$
\begin{aligned}
\left|\begin{array}{cc}
\left(0: A_{i} B_{i}^{2}-a_{i}-b_{i}\right)^{+} & (1)^{+} \\
(0)^{+} & -\Sigma^{+}\left(A_{i} B_{i}^{2}-a_{i}-b_{i}\right)^{-1}
\end{array}\right| \\
=-\Pi^{+}\left(A_{i} B_{i}^{2}-a_{i}-b_{i}\right) \Sigma^{+}\left(A_{i} B_{i}^{2}-a_{i}-b_{i}\right)^{-1},
\end{aligned}
$$

and the first result $\left[19\right.$, p. 363] is immediate. To prove the second result, let $\mathbb{Q}^{i}$ denote the simplex $\mathscr{Q}$ with the vertex $A_{i}$ replaced by $A_{+}, i \in \mathscr{G}$. Then the $i$ th weight of $A_{+}$with respect to $\mathbb{Q}$ is

$$
\frac{v_{n}\left(\mathscr{Q}^{i}\right)}{v_{n}(\mathscr{Q})}=\frac{v_{n}\left(\mathscr{Q}^{i}\right) v_{n}(\Re)}{v_{n}(\mathscr{Q}) v_{n}(\mathscr{B})}=\frac{d_{n}\left(\mathscr{Q}^{i}, \mathscr{B}\right)}{d_{n}(\mathbb{Q}, \mathscr{B})}
$$

via $[19$, p. 356]. Substituting and manipulating as before shows the numerator to be $-\Pi^{(i)}\left(A_{i} B_{i}^{2}-a_{i}-b_{i}\right)$ which is symmetric in $\mathscr{Q}$ and $\mathscr{G}$ and the proof is complete.

Since the medians of a simplex concur at the centroid we have the following result [25].

COROLlary 5.3. If the medians of simplex $\mathbb{Q}$ are perpendicular to the corresponding faces of simplex $\mathfrak{B}$, then the medians of $\mathscr{B}$ are perpendicular to the corresponding faces of $\mathcal{Q}$.

If $\mathscr{B}$ coincides with $\mathscr{Q}$ we have the following well-known result $[19$, p. 331], [17, p. 358], [3], [7].

COROLlary 5.4. Let $\mathbb{Q}$ be an orthocentric simplex with orthocenter $A_{+}$. Then there exist parameters $a_{i}, i \in \mathscr{G}_{+}$, such that $A_{i} A_{j}^{2}=a_{i}+a_{j}, i \neq j$, and $\Sigma^{+} a_{i}^{-1}$ $=0$.

In fact, to within an isometry, an orthocentric simplex has $n+1$ degrees of freedom (d.f.) and is determined by its parameters. However a pair of orthologic dupoints can be obtained by starting with an arbitrary $n$-simplex $\left(\frac{1}{2} n(n+1)\right.$ d.f.), choosing the point $B_{+}$arbitrarily in its space $(n$ d.f.) and locating $B_{i}$ arbitrarily on the perpendiculars from $B_{+}$to $\mathbb{Q}_{i}, i \in \mathcal{G},(n+1$ d.f.), so that the $2 n+2$ parameters cannot determine the dupoints.

We now extend the concept of orthologic simplexes to $m$-simplexes in $n$ space. The $(n-m+1)$-flats perpendicular to the $m$-flat of an $m$-simplex have an improper $(n-m-1)$-flat in common. If, in addition they have a proper point in common, then they have a proper $(n-m)$-flat in common and are coaxial. Two $m$-simplexes $\mathscr{Q}$ and $\mathscr{B}$ in $n$-space are said to be orthologic if the $(n-m+1)$-flats through $A_{i}$ and perpendicular to $\mathscr{B}_{i}, i \in g_{m}$, are coaxial; if 
$m=n$ this reduces to the original definition.

Theorem 5.2 is true in this more general setting once condition .2 is deleted. The proof that .1 implies .3 remains valid and it is not difficult to obtain a direct proof that .3 implies .1 using techniques similar to those used in the theorem below. In this result we consider conditions which turn out to yield a characterization of skew-orthologic simplexes.

THEOREM 5.5. Let $Q$ and $B$ be $m$-simplexes in $n$-space with centroids $A$ and $B$. For $0 \leqslant i<j \leqslant m$ let $\mathscr{F}_{i j}$ and $\mathscr{F}^{j}$ be primes perpendicular to edge $B_{i} B_{j}$ and passing through the midpoint of $A_{i} A_{j}$ and the centroid of the opposite $(m-2)$ face $\mathfrak{Q}_{i j}$ respectively, and let $\mathcal{S}_{i j}$ and $\mathcal{S}^{i j}$ be the primes perpendicular to $A_{i} A_{j}$ and passing through the midpoint of $B_{i} B_{j}$ and centroid of $\mathscr{B}_{i j}$ respectively. The following conditions are equivalent.

.1. One of the four sets of primes is coaxial, i.e. has an $(n-m)$-flat in common.

.2. All four sets of primes are coaxial.

.3. For all triples of distinct subscripts,

$$
t_{i j k}=\left(A_{i} B_{j}^{2}-A_{j} B_{i}^{2}\right)+\left(A_{j} B_{k}^{2}-A_{k} B_{j}^{2}\right)+\left(A_{k} B_{i}^{2}-A_{i} B_{k}^{2}\right)=0 .
$$

If the common flats are $\mathcal{P}, \mathcal{P}^{\prime}, \mathcal{Q}$ and $\mathcal{2}^{\prime}$, then $\mathcal{P}^{\prime}=\mathscr{P} D(A,-2 /(m-1))$ and $2^{\prime}=2 D(B,-2 /(m-1))$.

Proof. There is a proper point common to the $\mathscr{F}_{i j}$ if and only if the following system of $\left(\begin{array}{c}m+1 \\ 2\end{array}\right)$ equations has a solution:

$$
e_{i j}=\left[\mathbf{x}-\frac{1}{2}\left(\mathbf{a}_{i}+\mathbf{a}_{j}\right)\right] \cdot\left(\mathbf{b}_{i}-\mathbf{b}_{j}\right)=0, \quad 0 \leqslant i<j \leqslant m .
$$

Now, choosing any point as origin and using the identity (5.1) we see that

$$
\begin{aligned}
e_{i j}+e_{j k}+e_{k i}= & \frac{1}{2}\left(\mathbf{a}_{i} \cdot \mathbf{b}_{j}-\mathbf{a}_{j} \cdot \mathbf{b}_{i}\right)+\frac{1}{2}\left(\mathbf{a}_{j} \cdot \mathbf{b}_{k}-\mathbf{a}_{k} \cdot \mathbf{b}_{j}\right) \\
& +\frac{1}{2}\left(\mathbf{a}_{k} \cdot \mathbf{b}_{i}-\mathbf{a}_{i} \cdot \mathbf{b}_{k}\right) \\
= & -\frac{1}{4} t_{i j k},
\end{aligned}
$$

so the vanishing of $t_{i j k}$ is necessary for the solution of (5.2). It is also sufficient. In fact, (5.2) clearly has a solution if we restrict $i$ to the value zero. Since $e_{0 j}, e_{k 0}=e_{0 k}$ and $t_{i j k}$ vanish, it follows that $e_{j k}$ vanishes.

Further, $t_{i j k}$ is (skew-) symmetric in $\mathscr{Q}$ and $\mathscr{B}$ so 3 also implies that the $\mathcal{S}_{i j}$ are coaxial. Finally, the dilation $D(A,-2 /(m-1))$ sends the midpoint of $A_{i} A_{j}$ into the centroid of $\mathscr{Q}_{i j}$ and hence $\mathscr{F}_{i j}$ into $\mathscr{F}^{i j}, 0 \leqslant i<j \leqslant m$, and so $\mathscr{P}$ into $\mathcal{P}^{\prime}$. The result for 2 and $\mathcal{Q}^{\prime}$ is immediate which concludes the proof.

Some special cases of this result are known. For $m=2$ we have the case of orthologic triangles which in 3-space was considered by Mantel [13]. Interest- 
ingly, instead of the condition $t_{012}=0$, Mantel chose the origin on the common line and derived the multiplicative condition

$$
\left(a_{2} \cdot b_{1}\right)\left(a_{0} \cdot b_{2}\right)\left(a_{1} \cdot b_{0}\right)-\left(a_{1} \cdot b_{2}\right)\left(a_{2} \cdot b_{0}\right)\left(a_{0} \cdot b_{1}\right)=0 .
$$

The case $m=n$ for $n=3$ is given in [18, p. 3] and $\mathscr{Q}$ and $\mathscr{B}$ are said to be orthologic by the midpoints of the edges. The case when $m=n$ and $B$ coincides with $Q$ yields the well-known Monge Theorem [18, p. 6].

COROLlary 5.6. Let $Q$ be an $n$-simplex with centroid $G$ and circumcenter $C$. The primes $\mathcal{F}^{i j}$ perpendicular to the edge $A_{i} A_{j}$ and passing through the centroid of the oppposite face $\mathbb{Q}_{i j}, i \neq j$, concur in a point $M$ such that

$$
\overrightarrow{G M}=\frac{-2}{n-1} \overrightarrow{G C}
$$

Proof. The primes $\mathscr{F}_{i j}$ perpendicular to the edge $A_{i} A_{j}$ and passing through the midpoint of $A_{i} A_{j}$ obviously concur at $C$.

We are now ready for our criteria for skew-orthologic simplexes.

THEOREM 5.7. Let $\mathbb{Q}$ be an $n$-simplex and $\mathfrak{B}$ a (possibly degenerate) $n$-simplex in its space. The following are equivalent.

.1. $Q$ and $B$ are skew-orthologic.

.2. There exist constants $x_{i}, i \in \mathscr{G}$, such that $x_{i} \cos \left(\mathbb{Q}_{i}, \mathscr{G}_{j}\right)=x_{j} \cos \left(\mathbb{Q}_{j}, \mathscr{B}_{i}\right)$; i.e. $\left(\cos \left(\mathbb{Q}_{i}, \mathscr{B}_{j}\right)\right)$ is semisymmetric.

.3. There exist constants $x_{i}, i \in \mathscr{g}$, such that $x_{i}+A_{i} B_{j}^{2}=x_{j}+A_{j} B_{i}^{2}, i, j$ $\in$ g; i.e. $\left(\exp \left(A_{i} B_{j}^{2}\right)\right)$ is semisymmetric.

.4. For all triples of distinct subscripts,

$$
\left(A_{i} B_{j}^{2}-A_{j} B_{i}^{2}\right)+\left(A_{j} B_{k}^{2}-A_{k} B_{j}^{2}\right)+\left(A_{k} B_{i}^{2}-A_{i} B_{k}^{2}\right)=0 .
$$

.5. The corresponding 2-faces of $\mathfrak{Q}$ and $\mathfrak{B}$ are orthologic.

.6. $\mathcal{Q}$ and $\mathfrak{B}$ are orthologic by the midpoints of the edges.

Proof. We have seen that .1 and .2 are equivalent and by the preceding theorem it is clear that $.4, .5$ and .6 are equivalent. Clearly .3 implies .4 . Suppose .4 is true and let $x_{0}=0, x_{i}=A_{0} B_{i}^{2}-A_{i} B_{0}^{2}$. If $j \neq 0$,

$$
A_{j} B_{i}^{2}-A_{i} B_{j}^{2}=\left(A_{j} B_{0}^{2}-A_{0} B_{j}^{2}\right)+\left(A_{0} B_{i}^{2}-A_{i} B_{0}^{2}\right)=-x_{j}+x_{i},
$$

so .3 and .4 are equivalent. A simple calculation shows that .3 implies that $\left(d_{i j}\right)$ is symmetric, completing the proof of Theorem 5.1, which in turn implies .1 .

To complete the proof we show that .1 implies .5. Let $\mathscr{F}_{0}, \mathscr{F}_{1}, \mathscr{F}_{2}$ respectively be primes through $B_{0}, B_{1}, B_{2}$ perpendicular to edges $A_{1} A_{2}, A_{2} A_{0}, A_{0} A_{1}$, and 
for $i \in \mathscr{G}$, let $\mathscr{P}_{i}$ be the line from $B_{i}$ perpendicular the face of $\mathcal{Q}_{i}$ of $\mathcal{Q}$. It follows that the prime $\mathscr{F}_{i}$ contains the line $\mathscr{P}_{i}, i=0,1,2$. Now the $(n-2)$ flat $\mathcal{L}=\mathscr{F}_{0} \cap \mathscr{F}_{1}$ which meets $\mathscr{P}_{0}$ and $\mathscr{P}_{1}$, is completely perpendicular to $A_{0} A_{1} A_{2}$ and therefore meets $\mathscr{P}_{3}, \ldots, \mathscr{P}_{n}$ (in improper points). By .1 , the $\mathscr{P}_{i}, i \in \mathcal{G}$, are associated so $\mathcal{L}$ also meets $\mathscr{P}_{2}$ in the point $P \in \mathscr{P}_{2} \subset \mathscr{F}_{2} . P$ must be proper since the improper $(n-3)$-flat of $\mathcal{L}$ cannot contain the $n-2$ independent improper points of $\mathscr{P}_{3}, \ldots, \mathscr{P}_{n}$ and $\mathscr{P}_{2}$. Thus $\mathscr{F}_{0}, \mathscr{F}_{1}, \mathscr{F}_{2}$ are coaxial with axis $\mathcal{L}$, and the same is true for any triple of distinct subscripts and we are done.

The following analog of Theorem 2.3 was given by J. Bilo [24]: $\mathbb{Q}$ and $\mathscr{B}$ are skew-orthologic if and only if their equivectors are mutually orthogonal.

If $\mathscr{B}$ coincides with $\mathbb{Q}$, condition .4 is trivially satisfied so we have

COROLlary 5.8 [FACT 5.1]. The altitudes of a simplex are associated.

Combining Theorems 5.2 and 5.7 we obtain the following non-Euclideantype result.

Corollary 5.9. Let $\mathbb{Q}$ and $\mathscr{B}$ be $n$-simplexes in the same space. Then $\left(\cos \left(Q_{i}, \mathscr{B}_{j}\right)\right)$ is composite or semisymmetric according as $\left(\exp \left(A_{i} B_{j}^{2}\right)\right)$ is.

Our next application will be a generalization of the orthopole theorem: Let $\mathcal{Q}$ be a triangle, $\mathcal{L}$ a line, and $B_{i}$ the orthogonal projection of $A_{i}$ on $\mathcal{L}, i \in g_{2}$. Then the perpendiculars from $B_{i}$ on $\mathbb{Q}_{i}, i \in g_{2}$ are concurrent.

ThEOREM 5.10 ([10], [14]). Let $\mathbb{Q}$ be a simplex, $\mathcal{F}$ a prime, and for $i \in \mathcal{G}$, let $B_{i}$ be the orthogonal projection of $A_{i}$ on $\mathscr{F}$, and $C_{i}$ the circumcenter of $\mathscr{B}_{i}$.

.1. The perpendiculars from $B_{i}$ on $\mathbb{Q}_{i}, i \in \mathcal{G}$, are associated.

.2. The perpendiculars from $C_{i}$ on $\mathbb{Q}_{i}, i \in \mathcal{G}$, are concurrent.

Proof. $A_{i} B_{j}^{2}=A_{i} B_{i}^{2}+B_{i} B_{j}^{2}, i \neq j$. Clearly $t_{i j k}=0$ for all triples of distinct integers. To prove .2 , let $R_{i}$ be the circumradius of $\mathscr{B}_{i}$, so $A_{i} C_{j}^{2}$ $=A_{i} B_{i}^{2}+R_{j}^{2}, i \neq j$, and the result is immediate via Theorem 5.2.3.

The following result is believed to be new even for $n=2$ or 3 .

THEOREM 5.11. Let $\mathcal{Q}$ and $\mathscr{B}$ be n-simplexes, $\mathcal{F}$ a prime, and let "denote orthogonal projection on $\mathscr{F}$. The following are equivalent.

.1. The perpendiculars from $A^{\prime \prime}$ on $\mathfrak{B}_{i}, i \in \mathscr{G}$, are concurrent (associated).

.2. The perpendiculars from $B_{i}^{\prime \prime}$ on $\mathbb{Q}_{i}, i \in \mathcal{G}$, are concurrent (associated).

.3. If $n \geqslant 3$, the $(n-1)$-dimensional dupoints $A^{\prime \prime}{ }_{i}, i \in 9$, and $B^{\prime \prime}, i \in 9$, are orthologic (skew-orthologic).

Proof. We have $A_{i}^{\prime \prime} B_{j}^{2}=A^{\prime \prime}{ }_{i} B_{j}^{\prime 2}+B_{j}^{\prime \prime} B_{j}^{2}$ and $A_{i} B_{j}^{\prime 2}=A_{i} A_{i}^{\prime 2}+A_{i}^{\prime \prime} B_{j}^{\prime \prime 2}$. To prove the concurrent case, note that for all quadruples of distinct subscripts, 


$$
\begin{aligned}
A_{i}^{\prime \prime} B_{l}^{2} & -A_{i}^{\prime \prime} B_{k}^{2}+A_{j}^{\prime \prime} B_{k}^{2}-A_{j}^{\prime \prime} B_{l}^{2} \\
& =A_{i}^{\prime \prime} B_{l}^{\prime \prime 2}-A_{i}^{\prime \prime} B_{k}^{\prime \prime 2}+A_{j}^{\prime \prime} B_{k}^{\prime \prime 2}-A_{j}^{\prime \prime} B_{l}^{\prime \prime 2} \\
& =A_{i} B_{l}^{\prime \prime 2}-A_{i} B_{k}^{\prime \prime 2}+A_{j} B_{k}^{\prime \prime 2}-A_{j} B_{l}^{\prime \prime 2},
\end{aligned}
$$

and the result follows from Theorem 5.3.

To prove the associated case, note that for all triples of distinct subscripts

$$
\begin{aligned}
\left(A_{i}^{\prime \prime} B_{j}^{2}-A_{j}^{\prime \prime} B_{i}^{2}\right)+\left(A_{j}^{\prime \prime} B_{k}^{2}-A_{k}^{\prime \prime} B_{j}^{2}\right)+\left(A_{k}^{\prime \prime} B_{i}^{2}-A_{i}^{\prime \prime} B_{k}^{2}\right) \\
=\left(A_{i}^{\prime \prime} B_{j}^{\prime \prime 2}-A_{j}^{\prime \prime} B_{i}^{\prime \prime 2}\right)+\left(A_{j}^{\prime \prime} B_{k}^{\prime \prime 2}-A_{k}^{\prime \prime} B_{j}^{\prime \prime 2}\right)+\left(A_{k}^{\prime \prime} B_{i}^{\prime \prime 2}-A_{i}^{\prime \prime} B_{k}^{\prime \prime 2}\right) \\
=\left(A_{i} B_{j}^{\prime \prime 2}-A_{j} B_{j}^{\prime \prime 2}\right)+\left(A_{j} B_{k}^{\prime \prime 2}-A_{k} B_{j}^{\prime \prime 2}\right)+\left(A_{k} B_{i}^{\prime \prime 2}-A_{i} B_{k}^{\prime \prime 2}\right),
\end{aligned}
$$

and the result follows from Theorem 5.7. Similary we may prove

THEOREM 5.12. If $\mathfrak{B}$ is the reflection of $\mathscr{Q}$ in some flat, then $\mathfrak{Q}$ and $\mathscr{B}$ are skew-orthologic.

We conclude this section by examining what can be said if the matrix $\left(A_{i} B_{j}\right)$ is composite or skew-symmetric. A corner $A_{0}-A_{1} \cdots A_{n}$, the $n$-dimensional analog of an angle, is what remains of the simplex $\mathscr{Q}$ when the face $\mathbb{Q}_{0}$ is deleted. An isoclinal prime of a corner is a prime which makes equal angles with the edges of the corner and hence cuts equal segments on the edges.

THEOREM 5.13. Let $\mathbb{Q}$ and $\mathscr{B}$ be $n$-simplexes. For $i \in \mathcal{G}$, let $\mathcal{E}_{i}$ and $\mathscr{F}_{i}$ be the primes through $B_{i}$ respectively tangent to the circumsphere of

$$
B_{i} A_{i+1} \cdots A_{n} A_{0} \cdots A_{i-1}
$$

and isoclinal with the corner

$$
B_{i}-A_{i+1} \cdots A_{n} A_{0} \cdots A_{i-1}
$$

and let $\mathcal{S}_{i}$ and $\mathcal{H}_{i}$ be the primes through $A_{i}$ respectively tangent to the circumsphere of $A_{i} B_{i+1} \cdots B_{i-1}$ and isoclinal with the corner $A_{i}-B_{i+1} \cdots$ $B_{i-1}$. Then the following statements are equivalent.

.1. $Q$ and the simplex with faces $\mathcal{E}_{i}, i \in \mathcal{G}$, are perspective (associated).

.2. $\mathbb{Q}$ and the simplex with faces $\mathscr{F}_{i}, i \in \mathcal{G}$, are perspective (associated).

.3. $\mathscr{B}$ and the simplex with faces $\mathcal{G}_{i}, i \in \mathcal{G}$, are perspective (associated).

4. 9 and the simplex with faces $\mathcal{H}_{i}, i \in \mathfrak{G}$, are perspective (associated).

.5. The matrix of distances $\left(A_{i} B_{j}\right)$ is composite (semisymmetric).

Proof. Since a face of the tangential simplex is parallel to an antiparallel section for the corresponding corner, it follows from the proof of Fact 6.9 that the weights of $\varepsilon_{i}$ with respect to $B_{i} A_{i+1} \cdots A_{i-1}$ are $\left(B_{i} A_{j}^{2}: 0\right)$. It follows that the weights of $\mathscr{E}_{i}$ with respect to $Q$ are also $B_{i} A_{j}^{2}, j \neq i$, and the equivalence of 
.1 and .5 is immediate, and similarly for .3 and .5. Again, from the proof of Fact 6.8 it follows that the weights of $\mathscr{F}_{i}$ with respect to $B_{i} A_{i+1} \cdots A_{i-1}$ are $\left(B_{i} A_{j}: 0\right)$ and the equivalence of $.2, .4$, and .5 follows as before.

The primes tangent to the circumsphere of a simplex $\mathbb{Q}$ are the faces of the tangential simplex of $\mathbb{Q}$. The primes isoclinal to the corners of $\mathbb{Q}$ at the vertices contain the $\left(\begin{array}{l}n \\ 2\end{array}\right)$ external bisecting lines of the plane angles at the vertices and are the faces of the isoclinal simplex of $\mathcal{Q}$. If we let $\mathcal{Q}$ and $\mathscr{B}$ coincide in the preceding theorem we have the following known results.

Corollary 5.14 [FACT 6.5]. A simplex and its tangential simplex are associated.

Corollary 5.15 [21]. A simplex and its isoclinal simplex are associated.

6. Three dimensions versus higher dimensions. The reader is already aware of the considerable differences between the geometry of two dimensions and that of three dimensions. In this section we discuss some qualitative differences between the geometry of three dimensions and that of higher dimensions.

We recall our extensions of Pappus' theorem. Let $p$ be a permutation on $g$ and let $\mathscr{B}_{p}$ be the simplex with vertices $B_{0 p}, \ldots, B_{n p}$.

FACT 4.3. If $\mathscr{Q}$ and $\mathscr{B}$ are perspective and $\mathscr{Q}$ and $\mathscr{B}_{p}$ are perspective, then $\mathscr{Q}$ and $\mathscr{B}_{p^{k}}$ are perspective for every integer $k$.

FACT 4.4. If $\mathscr{Q}$ and $\mathscr{B}$ are associated and $\mathscr{Q}$ and $\mathscr{B}_{p}$ are associated, then $\mathscr{Q}$ and $\mathscr{B}_{p^{k}}$ are associated for every integer $k$.

We first observe that the proof of Facts 4.3 and 4.4 can be equally well applied to any of the matrices of Theorems 5.2 and 5.7 to yield

THEOREM 6.1. If $\mathscr{Q}$ and $\mathscr{B}$ are (skew-) orthologic and $\mathbb{Q}$ and $\mathfrak{B}_{p}$ are (skew-) orthologic, then $\mathbb{Q}$ and $\Re_{p^{k}}$ are (skew-) orthologic for every integer $k$.

A 2-dimensional version of Fact 4.3 and Theorem 6.1 is found in [22]. A close examination of our proof of Fact 4.3 shows that the following stronger result was actually proved.

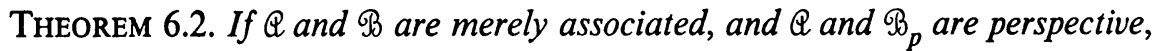
then $\mathscr{Q}$ and $\mathscr{B}_{p^{k}}$ are perspective for every integer $k$.

As strong as this result is, the full power of these seemingly mild hypotheses is not displayed for $n=2$ or 3 . In dimensions greater than the third, Berzolari [1] has shown that multiple perspectivity implies that $Q$ and $\mathscr{B}$ have some edges or vertices in common.

In the last result we had the help of a perspectivity. What can be done with associative relations alone? Let $p$ and $q$ be arbitrary permutations on $q, I$ the identity, and let $\{p\}$ denote the proposition: $\mathscr{Q}$ and $\mathscr{B}_{p}$ are associated. Fact 4.4 may be abbreviated to: $\{I\}$ and $\{p\}$ imply $\left\{p^{k}\right\}$ for all integers $k$. It follows that 
$\{p\}$ and $\{q\}$ do not imply $\{p q\}$ but rather $\left\{p q^{-1} p\right\}$ and $\left\{q p^{-1} q\right\}$. Berzolari [2, p. 246] has shown the following.

THEOREM. Let $A_{0}$ and $B_{0}$ be distinct and suppose we have $\{I\},\{p\}$ and $\{q\}$, where $p$ is the cycle $(01 \cdots n)$ and $q$ is the cycle $(1 \cdots n)$.

1. The number of associative relations generated is $n^{2}$.

2. If $n>3$, then $Q$ and $B$ are perspective (in $n^{2}$ ways).

3. If $n=3$, then $\mathscr{Q}$ and $\mathfrak{B}$ are mutually inscribed and circumscribed and for some numbers $a, b, c, d, e$, the vertices of $\mathfrak{B}$ are respectively proportional to $(0, a, b, c),(-a, 0, d, c e / a),(-b,-d, 0, c d / a),(-c,-c e / a,-c d / a, 0)$.

For tetrahedra $\mathscr{Q}$ and $\mathscr{B}$ which satisfy the weaker four-fold associative relations $\{I\},\{(01)(23)\},\{(02)(13)\},\{(03)(12)\}$, Jahnke [11] has shown that for some numbers $a, b, c, d, x, y, z$ the vertices of $\mathscr{B}$ are respectively proportional to $(a, b, c, d),(b, z a, y z d, c),(x c, x z d, z a, y d),(x y d, x c, y b, a)$. The special case of mutually self-polar tetahedra is obtained by taking $a=b=c=d, x=-y$ $=z=-1$.

Another difference between three and higher dimensions is given in [6, p. 44] where we remark that for $n=3$ there is a 1-parameter family of lines each of which is associated with three given lines, while for $n=4$ there is, in general, a unique line associated with four given lines, and for $n>4$ there is, in general, no line associated with $n$ given lines.

The proof of Fact 3.1 showed that if $n+1$ lines are associated, then there is an $(n-2)$-parameter family of secunda which meets them. For $n=3$ the lines of the one-parameter family are the rulers of a quadric surface and in 1827 Gergonne, Bobillier, Garbinsky and Steiner [8] showed that to prove four lines are associated it is sufficient to show that they are met by three lines. Because of this situation, many theorems have simple proof in three dimensions, such as those given in the references, which do not extend to higher dimensions. For $n>3$, Mandan [12] has shown that a necessary and sufficient condition for $n+1$ lines to be associated, i.e. that each point of each line has the property that through it passes an $(n-3)$-parameter family of secunda meeting all the lines, is that one point of each line have this property.

Sometimes the difference between three and higher dimensions are of an as yet unexplained nature. Call an $n$-simplex "special" if the lines from the vertices of $\mathscr{Q}$ to the circumcenter of the opposite faces are associated. Every triangle is special but for $n>3$, not every $n$-simplex is special. We pose the problem of finding an alternate characterization of such simplexes.

Since the orthocentric simplex is very often the correct generalization of the triangle to $n$-space, one may conjecture that "special" means orthocentric. However, even for $n=3$, this is only partially true for here "special" means "orthocentric, equifacial, or bisymmetric $\left(A_{0} A_{1}=A_{1} A_{2}=A_{2} A_{3}=A_{3} A_{0}\right)$ ". 
(See [9] which missed the bisymmetric case $S A=A B=B C=C S$ corresponding to $L=N, U=W=0$ in his system on p. 272.)

Now the proper analog of an equifacial tetrahedron is known only when $n$ is of the form $2^{k}-1$. In these dimensions a subset of the vertices of the analog of a cube can be the vertices of a regular simplex; the corresponding vertex subset of the analog of a rectangular parallelepiped is the vertex set of a special simplex. Our final theorem will show that it is a quirk of dimension 3 that an orthocentric simplex is special.

It is known [17, §27] [3] that the $\left(\begin{array}{c}n+1 \\ k+1\end{array}\right)$ centroids of the $k$-dimensional faces of an orthocentric simplex $\mathbb{Q}$ lie on a sphere, which we shall call the $k$-sphere. The 0 -sphere is the circumsphere; the center of the $\left(\frac{1}{2}(n-1)\right)$-sphere is the centroid of $\mathcal{Q}$.

THEOREM 6.3. The lines joining each vertex $A_{i}$ of an orthocentric simplex $\mathbb{Q}$ to the center $C_{i}$ of the $k$-sphere of the opposite face concur if $k=\frac{1}{2}(n-2)\left(\right.$ for $C_{i}$ is the centroid of the face) and are associated if $k=\frac{1}{2}(n-3)$. Unless $\mathbb{Q}$ is regular, these statements are false for any other value of $k$.

Proof. The weights of $C_{i}$ are given, in terms of the parameters of Corollary 5.4 (see the references), by

$$
\begin{aligned}
& 2(k+1) c_{i j}=1+(n-2 k-2) y_{i}^{-1} a_{j}^{-1}, \quad j \neq i, \\
& \text { where } y_{i}=a_{i}^{-1}-\sum a_{1}^{-1} .
\end{aligned}
$$

Clearly if $k=\frac{1}{2}(n-2)$ the matrix $\left(c_{i j}\right)$ is composite and if $k=\frac{1}{2}(n-3)$ then

$$
2(k+1) y_{i} c_{i j}=a_{i}^{-1}+a_{j}^{-1}-\sum a_{1}^{-1}=2(k+1) y_{j} c_{j i}, \quad i \neq j,
$$

while for other values of $k$ no multipliers exist unless the $a_{i}$ are equal.

We add that the representation in (6.1) admits the possibility of allowing $k$ to approach $\infty$. In this case the limiting sphere in the polar sphere of the face with its center $C_{i}=\left(y_{i} a_{j}^{-1}: 0\right)$ at the orthocenter and the lines $A_{i} C_{i}$ concur.

The author wishes to express his thanks to S. R. Mandan and Arun Sanyal for sharing with him their valuable insights in the form of personal correspondence and unpublished manuscripts, and to Donald McCarthy for suggesting improvements in the presentation.

\section{REFERENCES}

1. L. Berzolari, Sulla omologia di due piramidi in un iperspazio, Atti Accad. Naz. Lincei Rend. Cl. Sci. Fis. Mat. Natur. (5) 13 (1904), 446-451.

2. - Sui sistemi di $n+1$ rette dello spazio ad $n$ dimensioni situate in posizion di Schlafi, Rend. Circ. Mat. Palermo (2) 20 (1905), 229-247.

3. E. Egerváry, On orthocentric simplexes, Acta Litt. Sci. Szeged 9 (1940), 218-226. MR 1, 157.

4. R. W. Genese, Question 18229, Educational Times Reprints (3) 2 (1917), 15, 49-50. 
5. L. Gerber, Spheres tangent to all the faces of a simplex, J. Combinatorial Theory Ser. A 12 (1972), 453-456. MR 45 \#7607.

6. - Associated and perspective simplexes, Trans. Amer. Math. Soc. 201 (1975), 43-55. MR 50 \# 8262.

7. MR 51 \# 12717.

8. Proposed as a problem by J. D. Gergonne, Annales Math. 17 (1826-27), 83. Solved by E. E. Bobillier and Garbinsky, ibid. 18 (1827-28), 182-184, and by Steiner, Crelle 2 (1827), 268.

9. G. Glaeser, Sur une classe de tétraèdres, Rev. Math. Spéc. 62 (1951/52), 269-272.

10. R. Goormaghtigh, A generalization of the orthopole theorem, Amer. Math. Monthly 41 (1934), 440-441.

11. E. Jahnke, Aufgaben und Lehrsätze \# 104, Arch. Math. Phys. (3) 8 (1904-05), 81-82.

12. S. R. Mandan, Pascal's theorem in n-space, J. Austral. Math. Soc. 5 (1965), 401-408. MR 32 \#6276.

13. W. Mantel, Oplosung von Vraagstuk LXXVI, Wiskundige Opgaven 9 (1902-04), 168-173.

14. Proposed as Question 11933 and again as Question 16101 by J. Neuberg in Educational Times Reprints. Solved by A. Droz-Farny, ibid. 60 (1894), 54-55; by A. M. Nesbitt, ibid. (2) 12 (1907), 67; and by E. J. Nansen, ibid. (2) 13 (1908), 51.

15. J. Neuberg, Memoir sur le tétraèdre, Acad. Roy. Sci. Lettres Beaux-arts Belgique, Mem. Couronnes 37 (1884), 1-72.

16. - Sur les equicentres de deux systemes de $n$ points, Mem. Soc. Roy. Sci. Liège (3) 10 (1914), 15 pp.

17. W. J. C. Sharp, On the properties of simplicissima, Proc. London Math. Soc. 18 (1886/87), 325-359; ibid. 19 (1888), 423-482.

18. V. Thébault, Parmi les belles figures de géométrie dans l'espace, Librairie Vuibert, Paris, 1955. MR 16, 737.

19. G. Darboux, Sur les relations entre les groupes de points, de circles et de sphères dans le plan et dans l'espace, Ann. Ecole Norm. Sup. (2) 1 (1872), 323-392.

20. O. Dunkel (proposal and solution), Problem 3830, Amer. Math. Monthly 46 (1939).

21. N. A. Court (proposal) and O. Li (solution), Problem 4271, Amer. Math. Monthly 56 (1944), 420-421.

22. G. D. Simonesco, Sur les triangles trihomologique et triorthologique, Bull. Math. Phys. Ecole Polytech. Bucarest 6 (1936), 191-198.

23. J. Garfunkel and S. Stahl, The triangle reinvestigated, Amer. Math. Monthly 72 (1965), 12-20.

24. J. Bilo, Sur l'affinité orthologique, Mathesis 65 (1956), 509-516. MR 18, 592.

25. Moret-Blanc, Solution to Question 1460, Nouv. Annales Math. (3) 3 (1884), 484-487.

Department of Mathematics \& Computer Science, St. John's University, Jamaica, New YoRK 11439 